\title{
13 Methode zur Entwicklung von Prozessportalen
}

Rainer Alt, Marc A. Cäsar, Florian Leser, Hubert Österle, Thomas Puschmann, Christian Reichmayr, Rudolf Zurmühlen

13.1 Einleitung 258

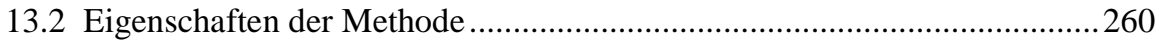

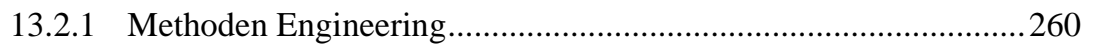

13.2.2 Analyse bestehender Methoden ............................................261

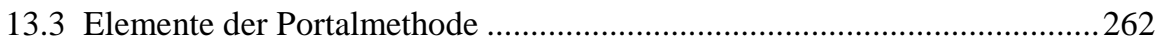

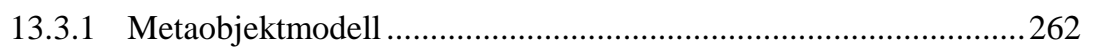

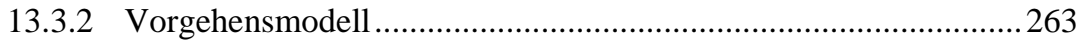

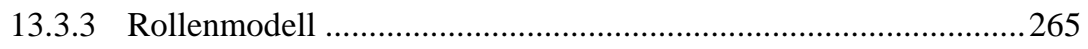

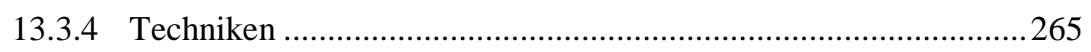

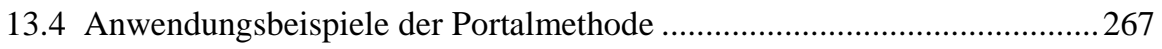

13.4.1 Potenzialanalyse bei Timecorp ................................................267

13.4.2 Kundenprozessanalyse und Portaldesign bei ETA SA ................2270

13.4.3 Kooperationsprozessanalyse bei ETA SA ................................2276

13.5 Zusammenfassung und Ausblick...........................................................2. 280 


\subsection{Einleitung}

Die in Kapitel 2 dargestellte Architektur zeigt Gestaltungselemente auf den drei Ebenen Strategie, Prozess und System. Zahlreiche Beispiele in den darauf folgenden Kapiteln haben die Anwendung dieser Architektur gezeigt. Um ein intuitives Vorgehen bei Entwurf und der Umsetzung von Echtzeit-Portalen zu vermeiden, sollen die bei den Fallbeispielen erzielten Erkenntnisse und Erfahrungen in ein systematisches Vorgehen einfliessen. Obgleich sich der Entwurf einer Architektur für das Echtzeit-Unternehmen niemals vollständig formalisieren lässt, können Methoden durch Modellbildung, Sequentialisierung der Problembearbeitung und die Auslagerung von Zwischen- und Endergebnissen in Dokumente die Entwicklungskomplexität verringern. Zwar kann der Erfolg eines Projektes auch damit nicht garantiert werden, aber zumindest lässt sich die Erfolgswahrscheinlichkeit und Wirtschaftlichkeit des Entwicklungsprozesses positiv beeinflussen [Hess 1996, 18f]. Für Portale erscheint der Methodeneinsatz aus mehreren Gründen sinnvoll:

- Die Gestaltung von Prozessportalen umfasst mehr als die reine Implementierung eines technischen Portalsystems. Wie in Kapitel 2 gezeigt, sind die Gestaltung von Geschäfts- und Prozessarchitektur von hoher Bedeutung. Die Entwurfskomplexität ist daher ähnlich wie beim Entwurf betrieblicher Prozesse (,Business Process Reengineering').

- Insbesondere in grossen Unternehmen entsteht eine Vielzahl an Portalen. Wie im Beispiel von Bosch gezeigt, verringert ein standardisiertes Vorgehen die Kosten eines wiederholten Portalentwurfs deutlich (s. Kap. 10.2.4). Bei Vorgabe standardisierter Lösungen erhöht sich ausserdem die Integrations- und Echtzeit-Fähigkeit des Unternehmens.

- In vielen Fällen entstehen Prozessportale nicht auf der ,grünen Wiese', sondern bauen auf vorhandenen Systemen im Kunden- und Lieferantenkontakt auf. Gerade Internetlösungen im Verkauf (,E-Commerce') und elektronische Marktplätze (s. Kap. 5, 8) werden nun um Leistungen aus dem Kundenprozess erweitert $^{49}$, um dadurch Kundennutzen und -bindung zu erhöhen. Dieses ,Reengineering' bestehender Systeme können Methoden unterstützen.

Zur systematischen Entwicklung von Methoden hat sich in der Wirtschaftsinformatik das ,Methoden Engineering' etabliert. Es liegt den verschiedenen Methoden der PROMET-Familie zugrunde, die sich in zahlreichen Projekten aus der Beratungspraxis bewährt haben. ${ }^{50}$ Verschiedene Inhalte dieser Methoden (z.B. die Dokumentation von Prozessen) lassen sich auch bei der Entwicklung von Prozess-

${ }^{49}$ Bei einer Untersuchung von 137 Produktkatalogen hatten nur 6\% eine Produktsuchfunktion, 4\% einen Seitenindex und 12\% eine Hilfefunktion. Weniger als 9\% hatten ,Frequently Asked Questions' (FAQ) und bei $47 \%$ fehlte eine interaktive Kommunikationsmöglichkeit über E-Mail [Lohse 1998, 83].

${ }^{50}$ PROMET (Prozessmethode) umfasst verschiedene Methoden zur Unterstützung von Transformationsprozessen von der Strategieentwicklung und Prozessgestaltung hin zur Systemimplementierung (www.promet-web.com). 
portalen nutzen. Die nachfolgende Methode soll gezielt die bisher nicht vorhandenen Inhalte erarbeiten. Es handelt sich dabei um:

- Die Portalstrategie mit der Positionierung des Prozessportals im Geschäftsnetzwerk, die Identifikation von Kundensegmenten und die Abschätzung der Portalpotenziale

- das Portaldesign mit der Analyse des Kundenprozesses, mit der Abbildung der Leistungen im Prozessportal sowie die Auslagerung von Aufgaben an externe Web Services,

- die Portalarchitektur mit den direkt und indirekt durch den Kundenprozess involvierten Applikationen und der Entwicklung der Komponenten einer übergreifenden Integrationsarchitektur.

Wie in Bild 13-1 gezeigt, ergänzt die nach den Prinzipien des ,Methoden Engineering' entwickelte Methode die Architektur des Echtzeit-Unternehmens. Nach einer Analyse bestehender Methoden in Kapitel 13.2 beschreibt Kapitel 13.3 die Elemente der Methode. Kapitel 13.4 zeigt die Anwendung ausgewählter Techniken anhand von zwei Fallbeispielen.

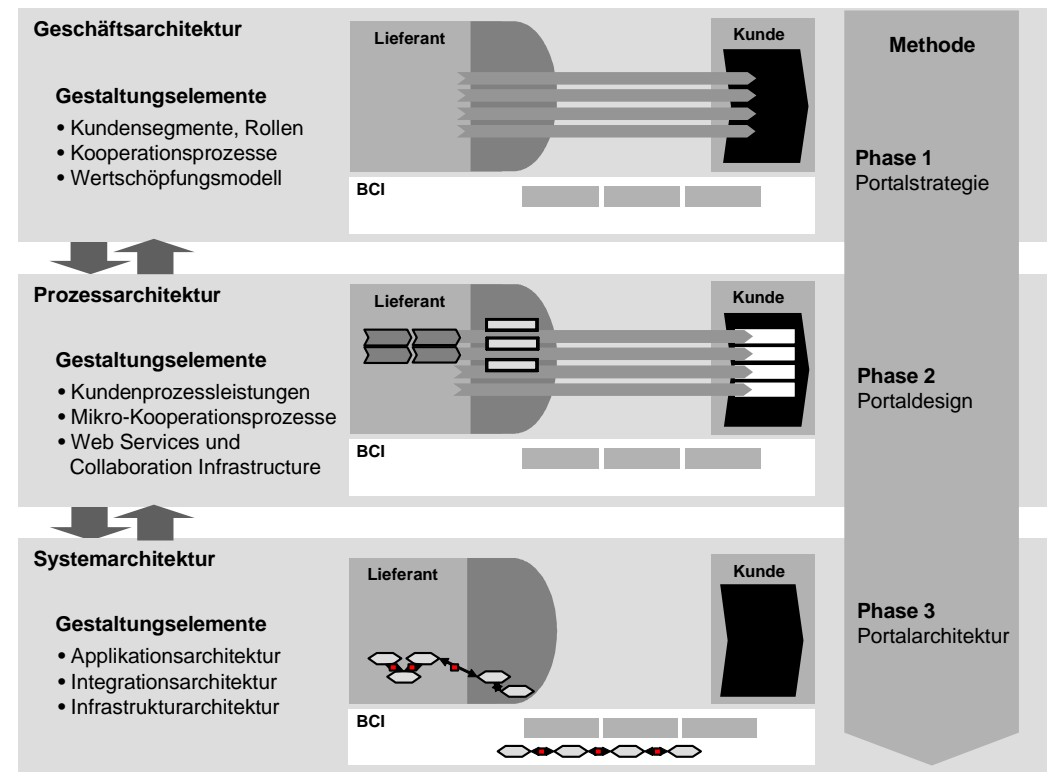

Bild 13-1: Architektur des Echtzeit-Unternehmens und Methode 


\subsection{Eigenschaften der Methode}

\subsubsection{Methoden Engineering}

Das ,Methoden Engineering' ist ein Ansatz zur systematischen Entwicklung von Vorgehensweisen und hat sich bereits mehrfach bewährt (vgl. [Legner 1999], [Pohland 2000]). Methoden umfassen danach fünf Bausteine [vgl. Gutzwiller 1994, 12ff] (s. Bild 13-2): ${ }^{51}$

- Ein Vorgehensmodell beinhaltet die empfohlene Reihenfolge der Aktivitäten auf oberster Ebene. Beispielsweise führt eine BPR-Methode von einer vorläufigen Analyse über das Makro-Design zum Mikro-Design eines Prozesses.

- Techniken beschreiben die Erarbeitung der Ergebnisse. Sie umfassen die nötigen Schritte, Metriken und zentralen Ergebnisdokumente. Werkzeuge wie das ARIS-Toolset können den Einsatz der Techniken unterstützen.

- Ergebnisdokumente halten die Resultate anhand bewährter Darstellungen, Tabellen und Grafiken fest. Ein Ergebnisdokument zur Analyse von IstProzessen ist beispielsweise die Prozessarchitektur.

- Rollen beschreiben, wer in einer bestimmten Phase an einem Projekt beteiligt sein sollte. Ausschlaggebend sind beispielsweise die zu treffenden Entscheidungen und das zur Erstellung der Ergebnisdokumente nötige Fachwissen.

- Das Metamodell beinhaltet die wesentlichen Gestaltungsobjekte und die Beziehungen zwischen diesen Bausteinen. So definiert eine BPR-Methode, dass Prozesse bestimmte Leistungen erzeugen und aus Aktivitäten bestehen.

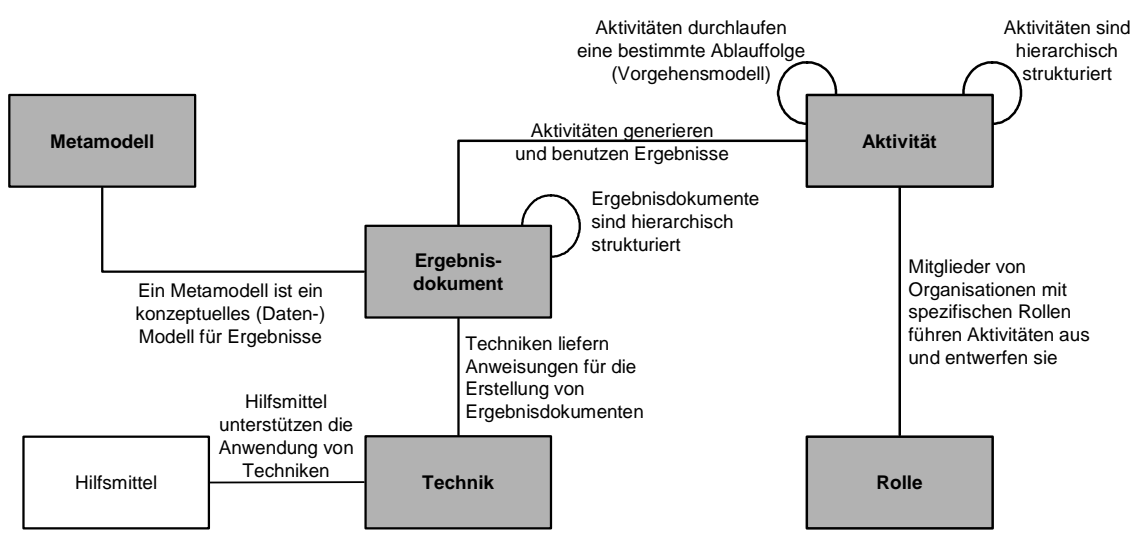

Bild 13-2: Elemente des Methoden Engineering [Gutzwiller 1994, 13]

${ }^{51} \mathrm{Zu}$ weiteren Beiträgen aus dem Methoden Engineering vgl. [Brinkkemper 1996], [Wetherbe 1994] und [Cutts 1988]. 


\subsubsection{Analyse bestehender Methoden}

In der Literatur existiert ein breites Spektrum an Forschungsgebieten, die spezifische Beiträge zur Entwicklung von Prozessportalen leisten können. Allerdings besitzen sie zwei wesentliche Defizite (s. Tabelle 13-1):

- Sie konzentrieren sich meist auf eine der Ebenen Geschäftsstrategie, Prozess und Informationssystem und bieten somit keinen durchgängigen Ansatz von der Portalstrategie bis zur Implementierung. Zwar zeigen PortaldesignMethoden auf, wie sich ein Portal technisch entwerfen lässt, sie vernachlässigen aber gleichzeitig die Kundenorientierung.

- Sie bieten häufig keine formalisierte Vorgehensweise im Sinne des ,Methoden Engineering'. Methoden zur Kundenprozessanalyse beinhalten oftmals ein Vorgehensmodell mit Techniken, aber weder Rollenmodell noch Ergebnisdokumente. Tabelle 13-2 zeigt dies anhand verschiedener Portalentwicklungsmethoden.

\begin{tabular}{|c|c|c|c|}
\hline & Stärken & Schwächen & Beispiele \\
\hline & \multicolumn{3}{|c|}{ Methoden zum Kooperationsmanagement } \\
\hline \multirow[t]{2}{*}{ 莺 } & 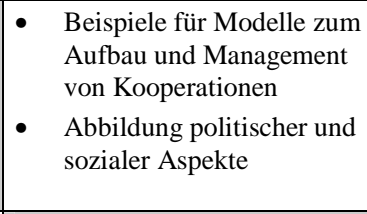 & 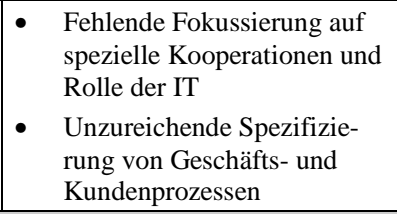 & $\begin{array}{l}\text { [Doz/Hamel 1998], } \\
\text { [Gomes-Casseres } \\
\text { 1996], } \\
\text { [Hakansson/Snehota } \\
\text { 1995], [Chisholm } \\
\text { 1998], [Sydow 1992] } \\
\end{array}$ \\
\hline & \multicolumn{3}{|c|}{ Methoden zum Business Process (Re-)Engineering } \\
\hline \multirow[t]{2}{*}{ 这 } & $\begin{array}{l}\text { - } \begin{array}{l}\text { Branchenübergreifend } \\
\text { anwendbare Methoden }\end{array} \\
\text { - } \quad \text { Spezielle Methoden (z.B. } \\
\text { für SCM oder CRM) mit } \\
\text { spezifischem Know-how }\end{array}$ & 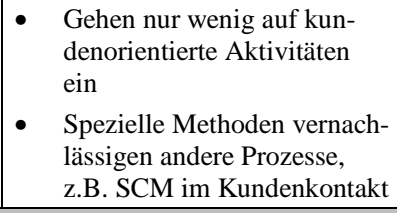 & $\begin{array}{l}\text { [Davenport 1993], } \\
\text { [Hess/Brecht 1996], } \\
\text { [Bowersox/Closs } \\
\text { 1996], [Christopher } \\
\text { 1998], [Kuglin 1998] }\end{array}$ \\
\hline & \multicolumn{3}{|c|}{ Methoden zur Kundenprozessanalyse } \\
\hline \multirow[t]{2}{*}{ 离 } & $\begin{array}{l}\text { Unterstützen Kundenpro- } \\
\text { zessanalyse aus verschie- } \\
\text { denen Perspektiven }\end{array}$ & $\begin{array}{l}\text { - } \quad \text { Theoretische Vorgehenswei- } \\
\text { sen ohne Handlungsanwei- } \\
\text { sungen } \\
\text { - Keine Ergebnisdokumente }\end{array}$ & $\begin{array}{l}\text { [Fleisch 1996], [de } \\
\text { Bakker/Seebacher } \\
\text { 2000], [Schulze 2002] }\end{array}$ \\
\hline & \multicolumn{3}{|c|}{ Methoden zum Portal-/Webdesign und zur IS-Implementierung } \\
\hline 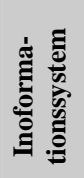 & $\begin{array}{ll}\text { - } & \text { Beinhalten wichtige } \\
\text { Anleitungen zur techni- } \\
\text { schen Implementierung } \\
\text { und zum Design }\end{array}$ & $\begin{array}{ll}\text { - } & \text { Starker Bezug auf System- } \\
\text { Anbieter und schwache Un- } \\
\text { terstützung auf Strategie- } \\
\text { und Prozessebene } \\
\text { - } & \text { Fokus auf interne Aspekte }\end{array}$ & $\begin{array}{l}\text { [Bayles 1998], [Tibco } \\
\text { 2001], [IMG 1998], } \\
\text { [Wen 2001] }\end{array}$ \\
\hline
\end{tabular}

Tabelle 13-1: Überblick zu relevanten Methoden für Prozessportale 


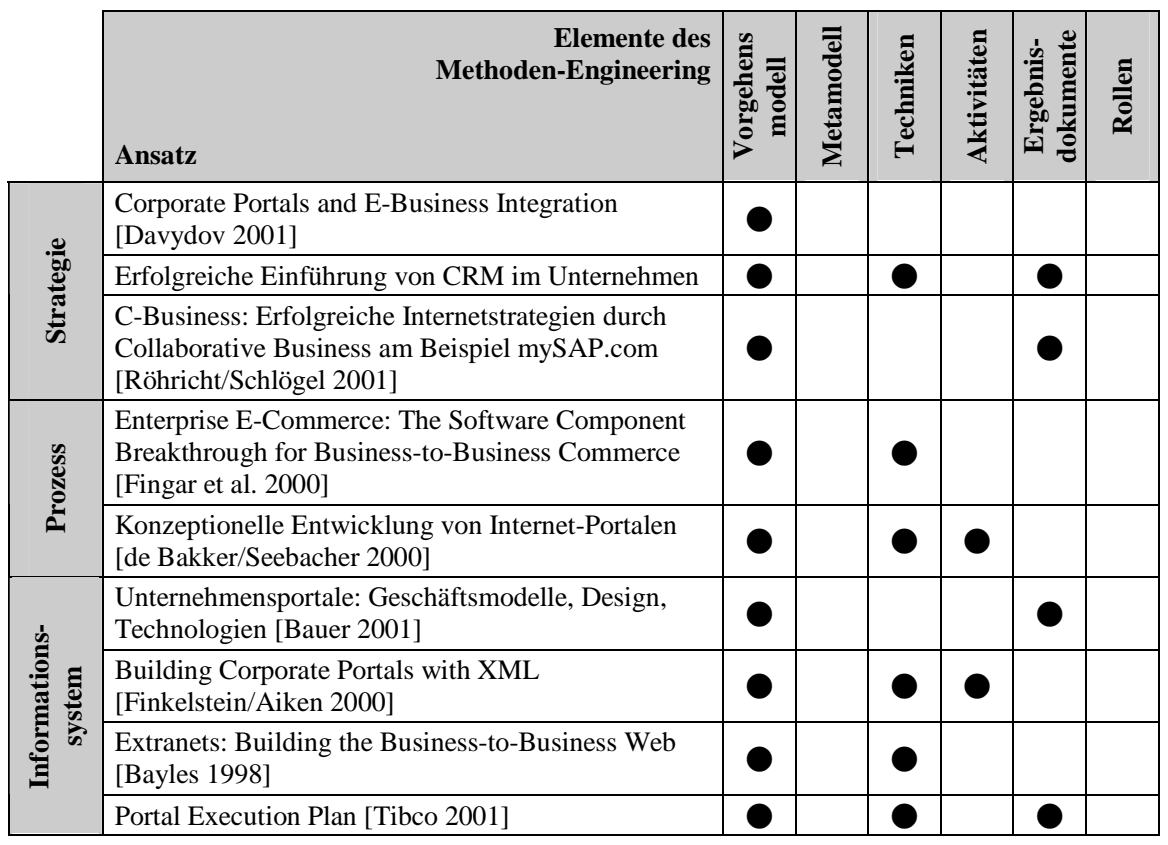

Tabelle 13-2: Vergleich bestehender Methodenansätze für Portale

\subsection{Elemente der Portalmethode}

Der folgende Abschnitt beschreibt eine Methode zur Entwicklung von Prozessportalen nach den Anforderungen des ,Methoden Engineering'. Vorausgesetzt wird, dass die strategischen Unternehmensziele definiert sind und die allgemeine Unternehmensstrategie die Entscheidung zum Aufbau eines Prozessportals enthält.

\subsubsection{Metaobjektmodell}

Ein Metaobjektmodell ist nach [Brenner 1995, 11] das konzeptionelle Datenmodell, welches das Ergebnis eines methodischen Vorgehens ist. Es dient dazu, die Konsistenz zu sichern und verschafft einen schnellen Überblick über die zu beschreibenden und gestaltenden Bereiche. Ferner ist es die Basis für einen Methodenvergleich [Legner 1999, 31ff]. Das vorliegende Metaobjektmodell baut auf den Ergebnissen von [Hess/Brecht 1996], [Riehm 1997], [Pohland 2000] und [Fleisch 2001b] auf. 


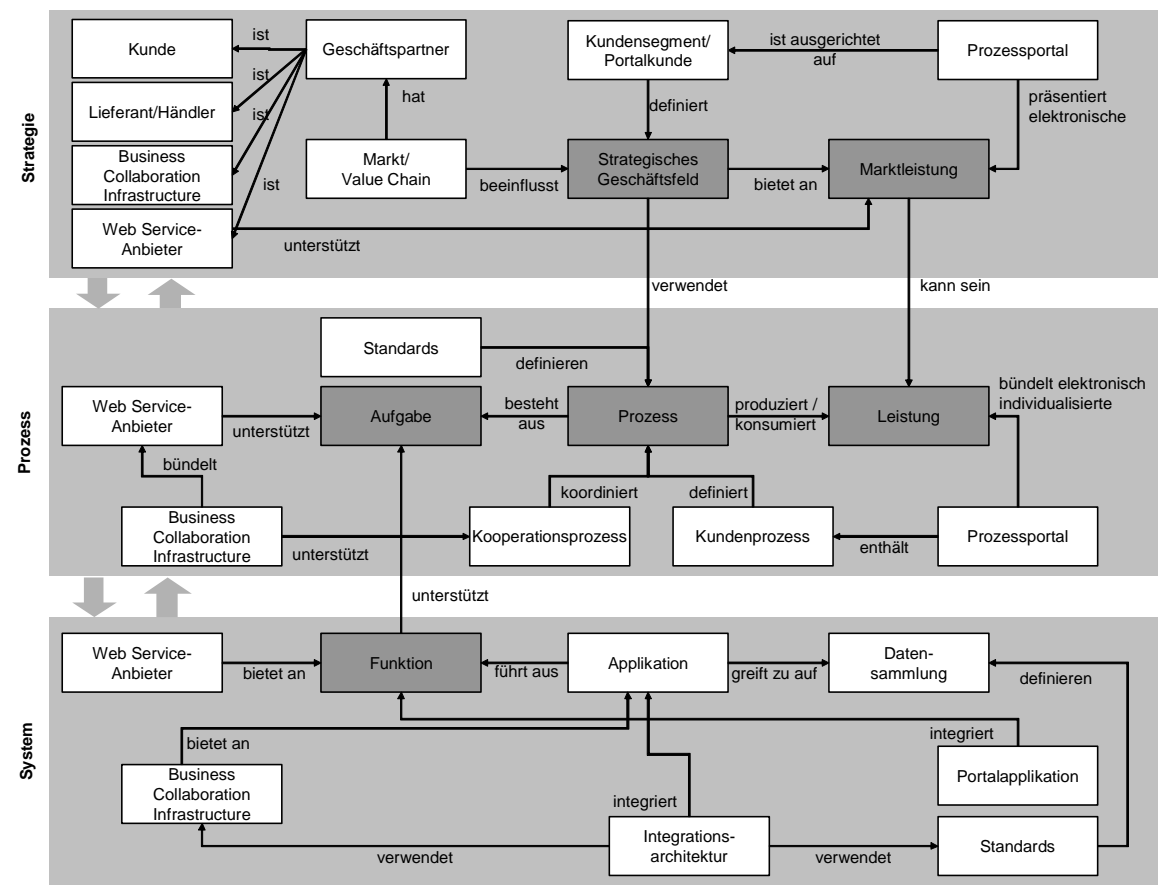

Bild 13-3: Metaobjektmodell der Business Networking-Methode

\subsubsection{Vorgehensmodell}

Das Vorgehensmodell umfasst die zeitlich logische Folge von Schritten in einem Problemlösungsprozess [Heinen 1991, 311]. Die einzelnen Phasen verbinden inhaltlich und logisch zusammenhängende Tätigkeiten, besitzen einen Start- und Endtermin, ein definiertes Abschlussergebnis und sehen am Ende eine ,Stop-orGo'-Entscheidung vor [Pohland 2000]. Das Vorgehensmodell zur Entwicklung von Prozessportalen besteht aus drei Phasen mit Techniken und Hauptergebnissen (s. Bild 13-4).

\section{Phase 1 (Portalstrategie)}

Die erste Phase umfasst die Analyse des Ist-Geschäftsnetzwerks sowie die Potenzialanalyse der möglichen Portalszenarien (Soll-Zustand). Im Rahmen dieser Untersuchung wird das gesamte Geschäftsnetzwerk mit den Kundensegmenten dargestellt (s. Kap. 2.2). Ausgehend von den Hauptleistungen und verschiedenen Prozess- und Portal-Erfolgsfaktoren lassen sich unterschiedliche Portalanbieter im Rahmen eines Benchmarks miteinander vergleichen. Anschliessend lassen sich daraus verschiedene Portalstrategien ableiten. Aus der Analyse der Portalstrategien folgen verschiedene Szenarien, die auf der Basis von Prozessverbesserungen, des potenziellen Portalnutzens (z.B. Kundenbindung) sowie mittels verschiedener ,Portalenabler' und der entstehenden Infrastrukturkosten bewertet werden. 
Phasenabschluss: Das Management des Projektinitiators entscheidet auf Basis der Potenzialanalyse, ob die nachgelagerten Projektphasen weiterverfolgt werden bzw. welches der Portalszenarien realisiert wird.

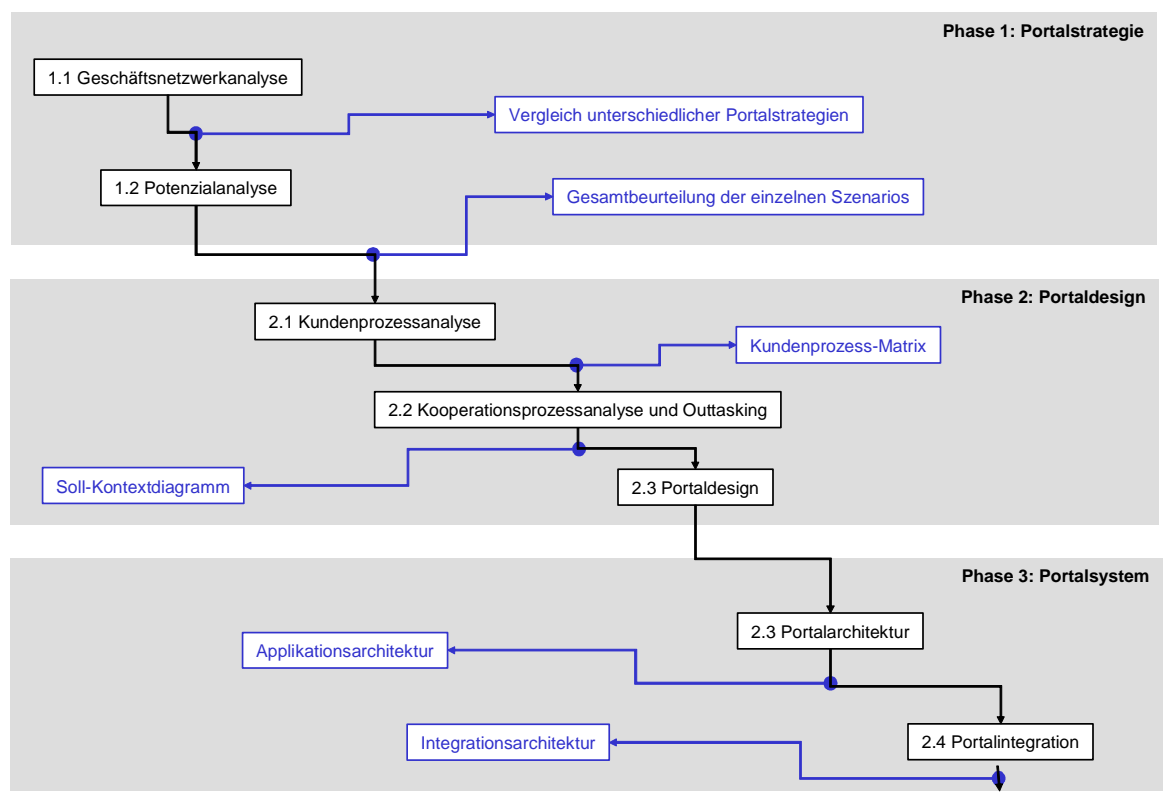

Bild 13-4: Techniken mit Abschlussergebnissen

\section{Phase 2 (Portaldesign)}

Aus der Kundenprozessanalyse lassen sich, ausgehend von den zu erreichenden Geschäftszielen, die ,Enabler' für das Prozessportal bestimmen. Dazu werden alle derzeit geschäftlich relevanten Aufgaben des Kunden, für die er vom Unternehmen Leistungen erwartet, erfasst, strukturiert und dokumentiert. Die Kooperationsprozessanalyse spiegelt die Kernkompetenzen des Unternehmens an einzelnen Aufgaben und bestimmt potenzielle Aufgaben, die sich auslagern lassen, sowie deren Kosten. Aufgaben, die das Unternehmen nicht selbst erledigen möchte, könen beispielsweise Web Service-Anbieter, die es zu evaluieren gilt, übernehmen. Die ausgewählten Dienste müssen dann in die Kooperationsprozessarchitektur des Unternehmens integriert werden. Der Schritt ,Portaldesign' ordnet einzelnen Kundengruppen Zugriffsrechte auf Portalinhalte oder -komponenten zu und definiert die Darstellung des Kundenprozesses im Prozessportal.

Phasenabschluss: Am Ende der Phase steht ein Konzept für das Design eines Prozessportals.

\section{Phase 3 (Portalsysteme)}

Für die Entwicklung der IS-Architektur gilt es zunächst, alle durch den Kundenprozess involvierten Applikationen festzulegen. Auf Basis der Soll-Appli- 
kationsarchitektur lässt sich die Architektur des Prozesspaketes definieren. Innerhalb der abschliessenden Portalintegration werden die für die Integrationsarchitektur relevanten Schnittstellen und Integrationsmuster bestimmt sowie Kriterien für die Auswahl von Portal- und Integrationswerkzeugen erhoben.

Phasenabschluss: Nach Abschluss der Phase liegen alle notwendigen Spezifikationen vor, um ein Prozessportal zu implementieren. Die Phase endet mit der Auswahl verschiedener Anbieter von Portal- und Integrationswerkzeugen.

\subsubsection{Rollenmodell}

Die Zuordnung von Aktivitäten zu Personen (Aufgabenträgern) wird als Rollenmodell bezeichnet. Eine Rolle kann von einer Geschäftseinheit oder einem einzelnen Mitarbeiter wahrgenommen werden. Die Aktivitäten sind mit Aufgaben, Kompetenzen und Verantwortung verbunden. Das Rollenmodell unterscheidet zunächst die bekannten Rollen der involvierten Mitarbeiter: Entscheider, Verantwortlicher und Unterstützer. Zusätzlich sind bei Kooperationsprojekten die Anwendungsmuster der Kunden und Partner miteinzubeziehen.

\begin{tabular}{|l|}
\multicolumn{1}{|c|}{ Rollen auf Ebene der Mitarbeiter } \\
\hline Entscheider $(E)$ : Personen oder Stellen, die Entscheidungen treffen können bzw. müssen. \\
\hline $\begin{array}{l}\text { Verantwortlicher }(V) \text { : Verantwortliche für das termingerechte Erreichen und das Weiterleiten von } \\
\text { Ergebnissen. }\end{array}$ \\
\hline $\begin{array}{l}\text { Unterstützer }(U) \text { : Personen, die inhaltliche Aussagen treffen können und im Sinne von Informations- } \\
\text { lieferanten in das Projekt einbezogen werden. }\end{array}$ \\
\hline \multicolumn{1}{|c|}{ Rollen auf Ebene der Geschäftseinheit } \\
\hline $\begin{array}{l}\text { Portalinitiator: Unternehmen, das massgeblich den Aufbau des Prozessportals geprägt und die } \\
\text { Kooperation initiiert hat. }\end{array}$ \\
\hline Portalkunde: Nutzer der durch das Prozessportal angebotenen Leistungen. \\
\hline $\begin{array}{l}\text { Web Service-Anbieter: Anbieter von Leistungen, welche das Unternehmen nicht selbst anbietet, aber } \\
\text { zur vollständigen Abdeckung des Kundenprozesses in das Portal integriert. }\end{array}$ \\
\hline
\end{tabular}

Bild 13-5: Rollenmodell auf Ebene von Mitarbeitern und Geschäftseinheiten

In Anlehnung an die ,inter-Business Networking-Methode' [Alt et al. 2002] geht eine Kooperationsidee von demjenigen aus, der das Portal initiiert und wird sukzessive konkretisiert. In der Regel formuliert das initiierende Unternehmen den Handlungsbedarf und schätzt die Potenziale ab. Im zweiten Schritt eines Portalprojekts werden Klienten und Web Service-Anbieter in das Projekt eingebunden, damit eine Win-win-Situation für alle Betroffenen entstehen kann.

\subsubsection{Techniken}

Die drei Phasen des Vorgehensmodells beinhalten verschiedene Techniken, welche das Erreichen von Ergebnisdokumenten konkretisieren. Bild 13-6 zeigt die wesentlichen Schritte der Techniken mit den wichtigsten Ergebnisdokumenten. 


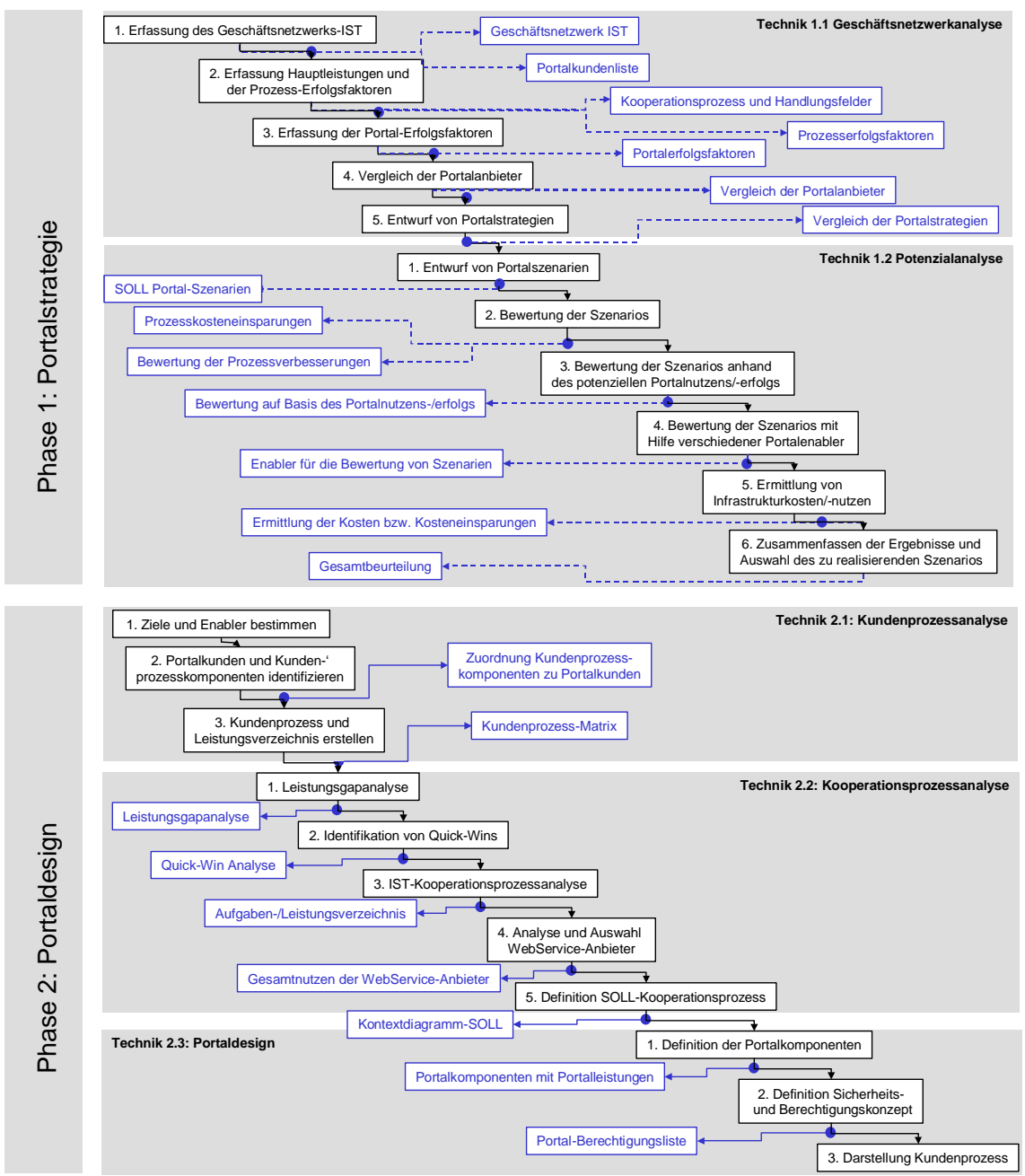

Bild 13-6: Überblick über Schritte und Ergebnisdokumente der Techniken (1) 


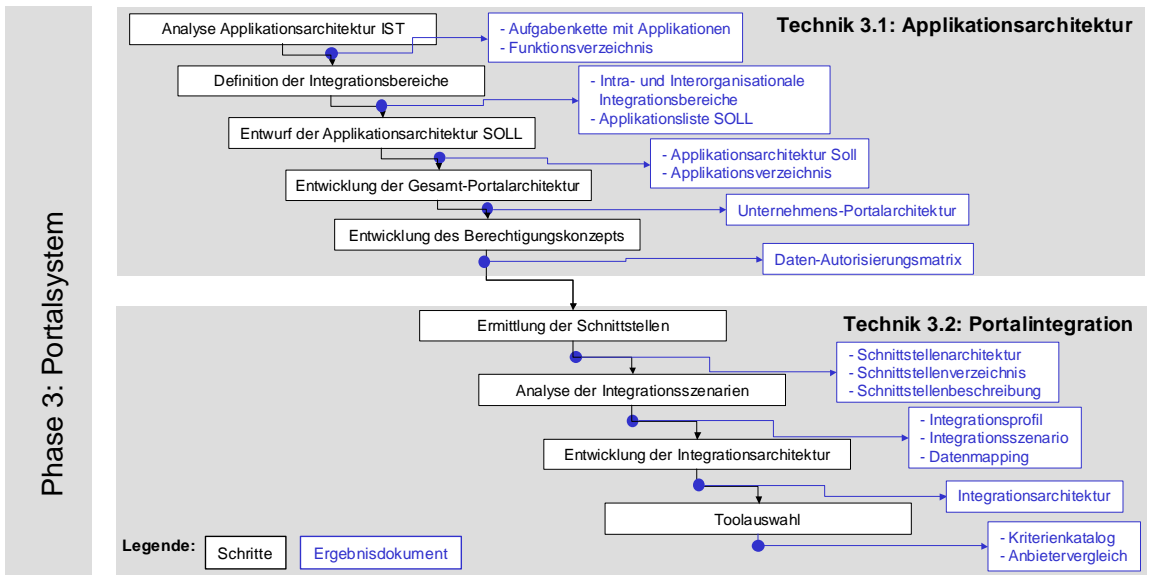

Bild 13-6: Überblick über Schritte und Ergebnisdokumente der Techniken (2)

\subsection{Anwendungsbeispiele der Portalmethode}

\subsubsection{Potenzialanalyse bei Timecorp}

Ausgehend von der vorhandenen Geschäftsarchitektur werden mit Technik 1.2 beim global agierenden Uhrenhersteller Timecorp AG künftige Portalszenarien erarbeitet und verglichen. Die Konzerngruppe besteht aus individuellen Firmen, die sich unter anderem auf Uhren, Ersatzteile sowie Forschung und Entwicklung spezialisiert haben. Im Folgenden wird die Ersatzteillogistik näher betrachtet (s. Bild 13-7). Geschäftskunden bestellen Ersatzteile sowohl bei (1) den Landesgesellschaften, (2) den einzelnen Marken und (3) bei Ersatzteillieferanten (nur Ersatzteile für Uhrwerke). Landesgesellschaften bestellen entweder bei der (4) Marke oder beim (5) Ersatzteillieferanten. Die Marken ordern Werkersatzteile wiederum bei (6) Ersatzteillieferanten. Bestellt wird dabei hauptsächlich per Fax und Brief, vereinzelt per EDI und bei Werkersatzteilen auch über den elektronischen Bestellweg des Ersatzteillieferanten. In der Regel folgt der Warenfluss dem Bestellfluss, d.h. Direktlieferungen von Ersatzteilen an Geschäftskunden sind die Ausnahme. Jedes Ersatzteilpaket wird vom Versender einzeln in Rechnung gestellt.

Ziel ist es, im Rahmen eines Workshops maximal drei realistische Fälle zu beschreiben, die in den nachfolgenden Schritten bewertet werden. Bei der Beschreibung der Soll-Abläufe sind alle Partner und Kunden innerhalb der Wertschöpfungskette, der Waren- sowie der Informations- und Bestellfluss zu berücksichtigen. 


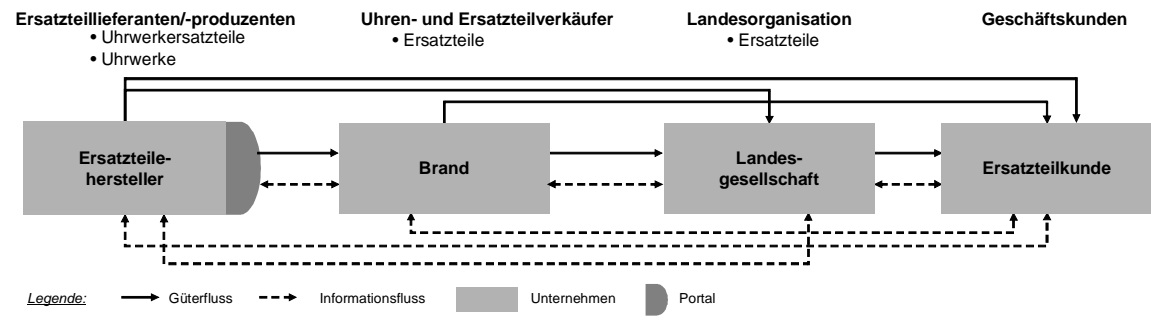

Bild 13-7: Geschäftsnetzwerk-IST bei Timecorp

Im Soll-Szenario 1 entwickelt jede Uhrenmarke ein eigenes Portal inklusive EShop-Lösung mit eigenem Web-Auftritt. Vorgaben der zentralen IT-Organisation müssen dabei berücksichtigt werden wie etwa einheitliche Prozessstandards, Schnittstellen und zentrale Stammdatenverwaltung. Bild 13-8 zeigt, dass eine Ersatzteilbestellung nach Eingang über ein Marken-Portal elektronisch nach Artikelverantwortlichkeiten gesplittet wird. Die beteiligten Unternehmen aktualisieren ihre Stammdaten nach bestimmten Regeln in einem zentralen Konzernstammdaten-Server. Die Marke, die den Kundenauftrag erhalten hat, erstellt eine Gesamtrechnung. Werkersatzteile werden vom Ersatzteillieferanten monatlich via Sammelrechnung oder im Gutschriftverfahren in Rechnung gestellt.

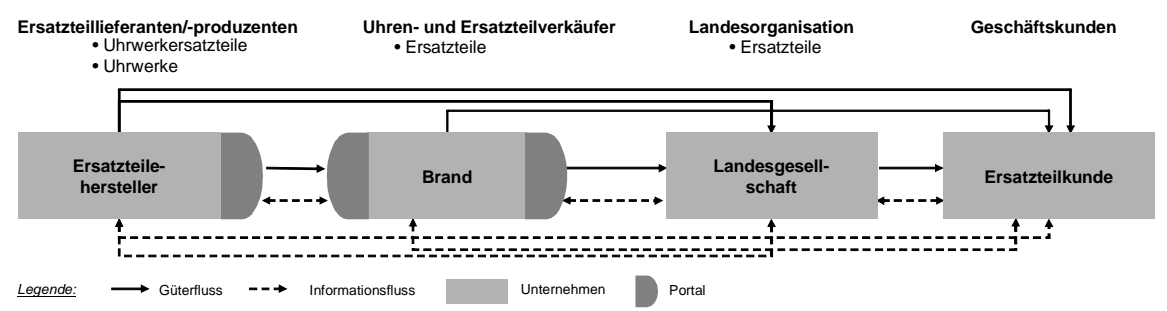

Bild 13-8: Soll-Portalszenario 1

Das Soll-Portalszenario 2 sieht ein zentrales Kundendienst-Portal für den gesamten Konzern vor (s. Bild 13-9). Jedes Konzernunternehmen erhält die Möglichkeit, seinen Internet-Auftritt in einem gewissen Rahmen selbst zu gestalten. Die zugrunde liegenden Prozesse wie Katalogfunktionen, Bestellablauf, Bezahlung und Verfügbarkeitsprüfung sowie die technische Lösung sind aber für die Konzerneinheiten standardisiert. Auch hier erfolgt nach der Ersatzteilbestellung über das Service-Portal eine elektronische Aufteilung der Orders nach Artikelverantwortlichkeiten. Die beteiligten Unternehmen halten ihre Stammdaten im zentralen Konzernstammdaten-Server aktuell nach Regeln, die es zu spezifizieren gilt (s. Szenario 1). Rechnungsstellung und Warenfluss entsprechen Szenario 1. 


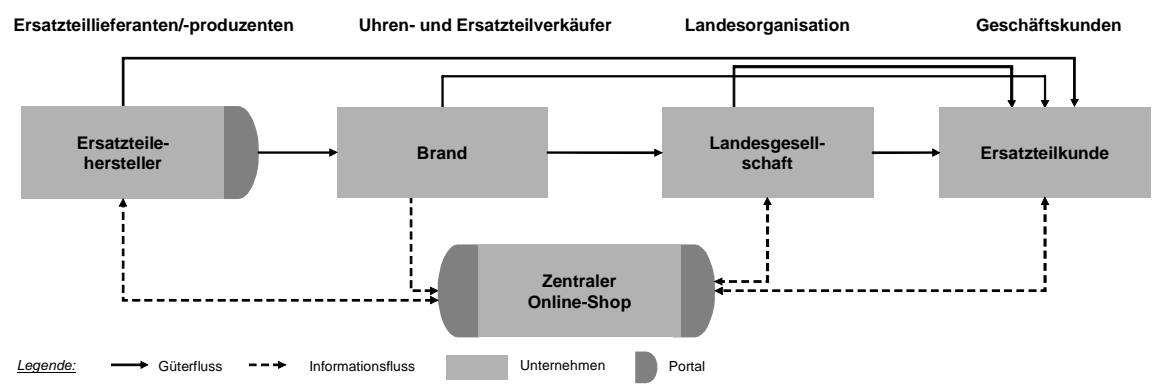

Bild 13-9: Soll-Portalszenario 2

Das Soll-Portalszenario 3 sieht gegenüber Szenario 2 ein zentrales Distributionszentrum vor, das alle Teillieferungen sammelt (s. Bild 13-10). Einzelbestellungen werden wie bisher versandt. Sind alle Teillieferungen, die zu einem Auftrag gehören, eingetroffen, wird eine Gesamtlieferung mit allen Ersatzteilen an den Kunden ausgelöst.

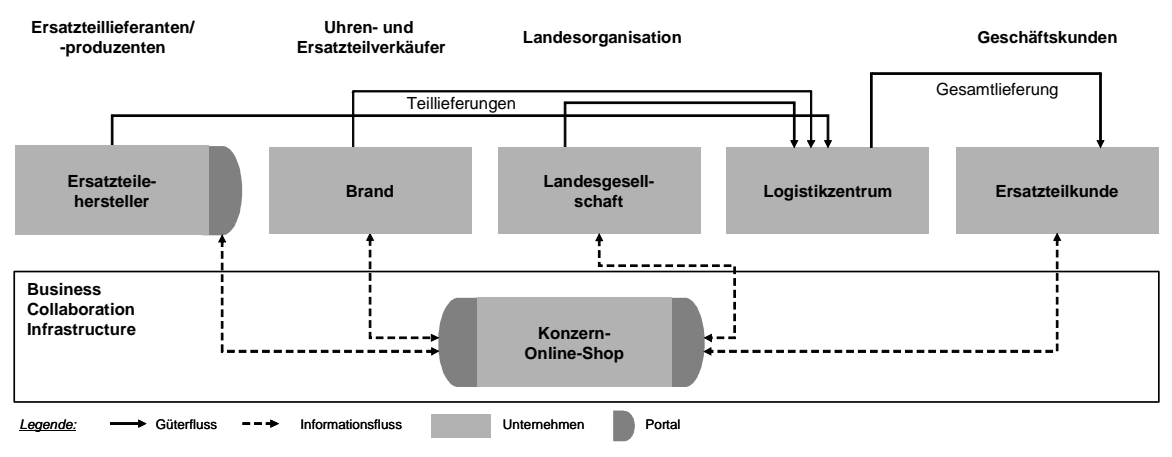

Bild 13-10: Soll-Portalszenario 3

Jedes Szenario hat spezifische Nutzeffekte für die Timecorp AG. Die Methode detailliert schrittweise den Nutzen der Szenarien nach den erreichbaren Prozesseffizienzen, den geschätzten Kundenbindungseffekten und den Rahmenbedingungen für die Realisierung (,Portalenabler'). Bis auf den Prozessnutzen erfolgt die Quantifizierung nicht monetär, sondern mittels einer Nutzwertanalyse. Die Infrastrukturkosten und Prozesseffizienzen werden monetär bewertet (s. Tabelle 13-3). Nach der Beurteilung der drei Alternativen entscheidet sich die Timecorp AG für das erste Szenario, da kurz- bis mittelfristig mit einer unmittelbaren Beteiligung mehrerer Uhrenmarken nicht gerechnet wird. Ist das Projekt erfolgreich, sollen Szenarios 2 und 3 nochmals neu bewertet werden. 


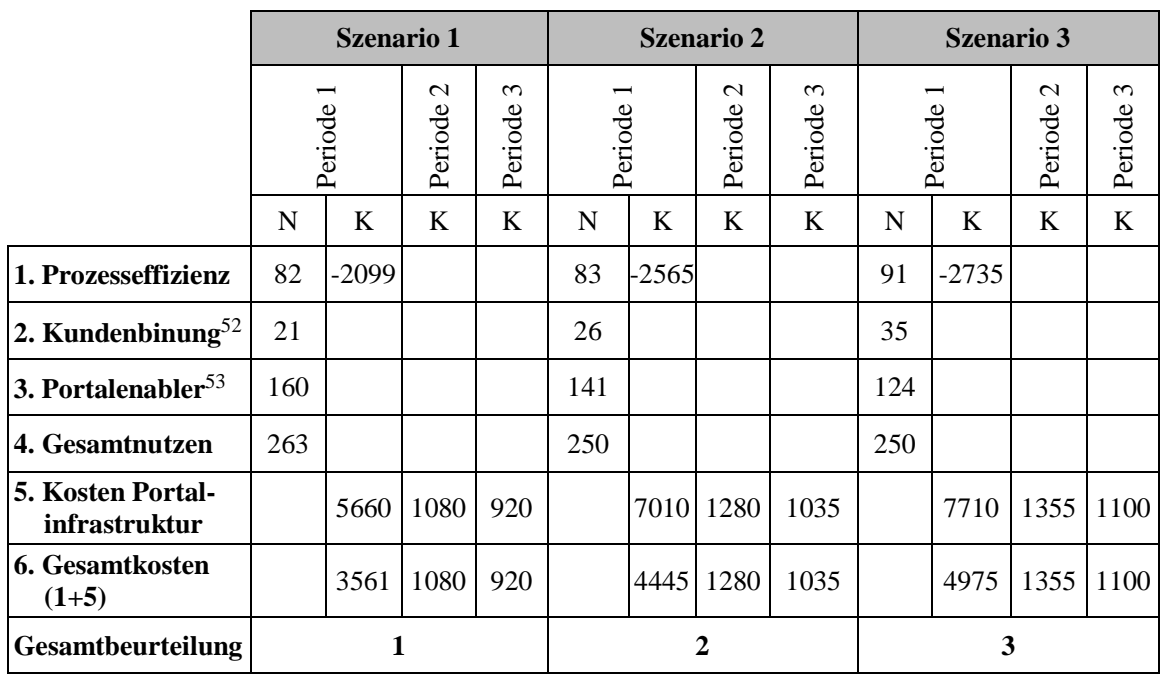

Legende: $\mathrm{N}=$ Nutzen, $\mathrm{K}=$ Kosten

Tabelle 13-3: Gesamtbeurteilung der einzelnen Szenarien (Kosten in Tausend CHF)

\subsubsection{Kundenprozessanalyse und Portaldesign bei ETA SA}

Der Wunsch, sich stärker an den Kundenbedürfnissen zu orientieren, die Kosten zu senken, Aufträge schneller zu bearbeiten und als globaler ,Player' aufzutreten, war bei ETA SA (s. Kap. 2.1.4) im Jahr 1998 Ausgangspunkt für die ersten ECommerce-Aktivitäten. Diese hatten primär das Ziel, die internen EchtzeitAnforderungen im Kundendienst (ETA-CS) zu verbessern. Ergebnis der ersten Phase (1996-1998) war der ETA Online Shop (EOS), der im Dezember 1999 startete. Obwohl sich der EOS klar am Kundenprozess von Uhrwerkersatzteilkunden orientiert, deckt er ihn bisher nicht vollständig ab. Er unterstützt den Kunden vor allem während des Einkaufsprozesses von Uhrwerkersatzteilen. Wichtige Probleme, die er vor und nach dem Einkauf hat, berücksichtigt der EOS aber bislang nicht. $\mathrm{Zu}$ den ungelösten Aufgaben gehören die Identifikation, welches Ersatzteil überhaupt benötigt wird, oder die Reparatur oder Wartung von Uhrwerken. Funktionen wie ,Order Tracking', , Repair Tracking' oder ,Parcel Tracking' sind deshalb entwickelt worden und liegen seit Dezember 2001 in der Version 3.0 des EOS vor. Zur Weiterentwicklung des EOS zu einem Portal, das Leistungen zum gesamten Kundenprozess in Echtzeit bereit stellt, hat ETA die Techniken 2.1 und 2.3 angewendet.

52 Indikatoren: Personalisierbarkeit, Differenzierung gegenüber Wettbewerbern, Kundenprozessabdeckung (s. Tabelle 2-2). Berechnungsbeispiel: Personalisierbarkeit (3 Punkte von 3 , Gewichtung $4=12$ Punkte).

${ }^{53}$ Indikatoren s. Tabelle 13-4. Berechnungsbeispiel: Übereinstimmung mit strategischen Geschäftsaussagen (3 Punkte von 3, Gewichtung $6=18$ Punkte). 
Die Ausgangsbasis der Weiterentwicklung sind die strategischen Geschäftsziele und die ,Portalenabler' (Treiber). Wie bereits erwähnt, sind ,Portalenabler' Rahmenbedingungen mit Einfluss auf die Gestaltung von Portal und Kooperationsprozessen (s. Tabelle 13-4). ${ }^{54}$ Wichtigste Ergebnisse für ETA-CS waren ein professioneller Auftritt gegenüber den Kunden, eine höhere Transparenz der Prozesse und die Betreuung der Informationssysteme durch interne Ressourcen.

\begin{tabular}{|c|l|}
\hline Portalenabler & \multicolumn{1}{c|}{ Beschreibung } \\
\hline $\begin{array}{c}\text { Geschäfts-/ } \\
\text { Vernetzungstreiber }\end{array}$ & $\begin{array}{l}\text { Fassen betriebswirtschaftliche Faktoren zusammen, welche die Portalar- } \\
\text { chitektur und die Kooperationsprozesse bestimmen, z.B. Übereinstim- } \\
\text { mung mit strategischen Geschäftsaussagen, Flexibilität des Unterneh- } \\
\text { mens. }\end{array}$ \\
\hline Politiktreiber & $\begin{array}{l}\text { Beziehen sich auf die Akzeptanz einer geplanten Portalarchitektur und } \\
\text { der Kooperationsprozesse vor dem Hintergrund der Interessen jedes } \\
\text { beteiligten Unternehmens / Partners, z.B. Widerstand gegen Veränderung, } \\
\text { Wunsch nach Einmaligkeit. }\end{array}$ \\
\hline Systemtreiber & $\begin{array}{l}\text { Konzentrieren sich auf die funktionellen Rahmenbedingungen für eine } \\
\text { zukünftige Portalarchitektur, z.B. realisierte Funktionalität, Modifikati- } \\
\text { onsbedarf. }\end{array}$ \\
\hline Techniktreiber & $\begin{array}{l}\text { Bestimmen die technischen Rahmenbedingungen aktueller und zukünfti- } \\
\text { ger Portale, z.B. Schnittstellen, Skalierbarkeit. }\end{array}$ \\
\hline Projekttreiber & $\begin{array}{l}\text { Verstehen sich als Realitätscheck aus der Implementierungsperspektive. } \\
\text { Sie überprüfen, ob ein Projektportfolio umsetzbar ist, z.B. bezüglich } \\
\text { Projektkomplexität und Ressourcenbegrenzungen. }\end{array}$ \\
\hline
\end{tabular}

Tabelle 13-4: Allgemeine Portalenabler

Zur Bestimmung der Kundenprozesse werden die wichtigsten Portalkunden mit einbezogen. Portalkunden sind in diesem Fall Anspruchsgruppen und -personen, die einen unmittelbaren Einfluss auf den Projektfortschritt haben und/oder von den Projektzielen direkt oder indirekt betroffen sind [vgl. IMG 1997, 126f]. Als Portalkunden des ETA-CS wurden ,Component Customer', ,Business Unit', ,Interessent' und ,Mitarbeiter' identifiziert.

Für jeden Kunden gilt es, die individuellen Leistungen, Bedürfnisse und Erwartungen an die Unternehmung bzw. Leistungen zu bestimmen, die das Portal individuell bereitstellen soll. Die Ergebnisse werden dann gemeinsam von den Teilnehmern gruppiert und ergeben so die für die Abbildung des Kundenprozesses benötigten Komponenten (s. Bild 13-11). Diese bilden gleichzeitig ein erstes Ordnungsraster für die zukünftigen Portalkategorien.

Zur Ableitung des Kundenprozesses wird die ,klassische' Prozessanalyse erweitert, die, ausgehend von einem groben Überblick, die wenigen, wettbewerbsentscheidenden Prozesse in Kontextdiagrammen verfeinert [Österle 1995, 61]. Ein für jeden Portalkunden erstelltes Kundenprozess-Kontextdiagramm führt innerhalb kürzester Zeit zu guten Übersichts- und Grobergebnissen. Der Vorteil liegt in der schrittweisen Analyse der Prozesse, Leistungen, zusätzlichen Prozessbeteilig-

${ }^{54}$ Vgl. dazu die Dimensionen der Netzwerkfähigkeit [Fleisch 2001, 207ff] und die Treiber der Applikationsarchitektur [Pohland 2000, 138ff]. 
ten und betroffenen IS in einem einzigen Vorgang. Die eigentliche Entwicklung des Kundenprozess-Kontextdiagramms besteht aus vier Schritten (s. Bild 13-12 und Tabelle 13-5):

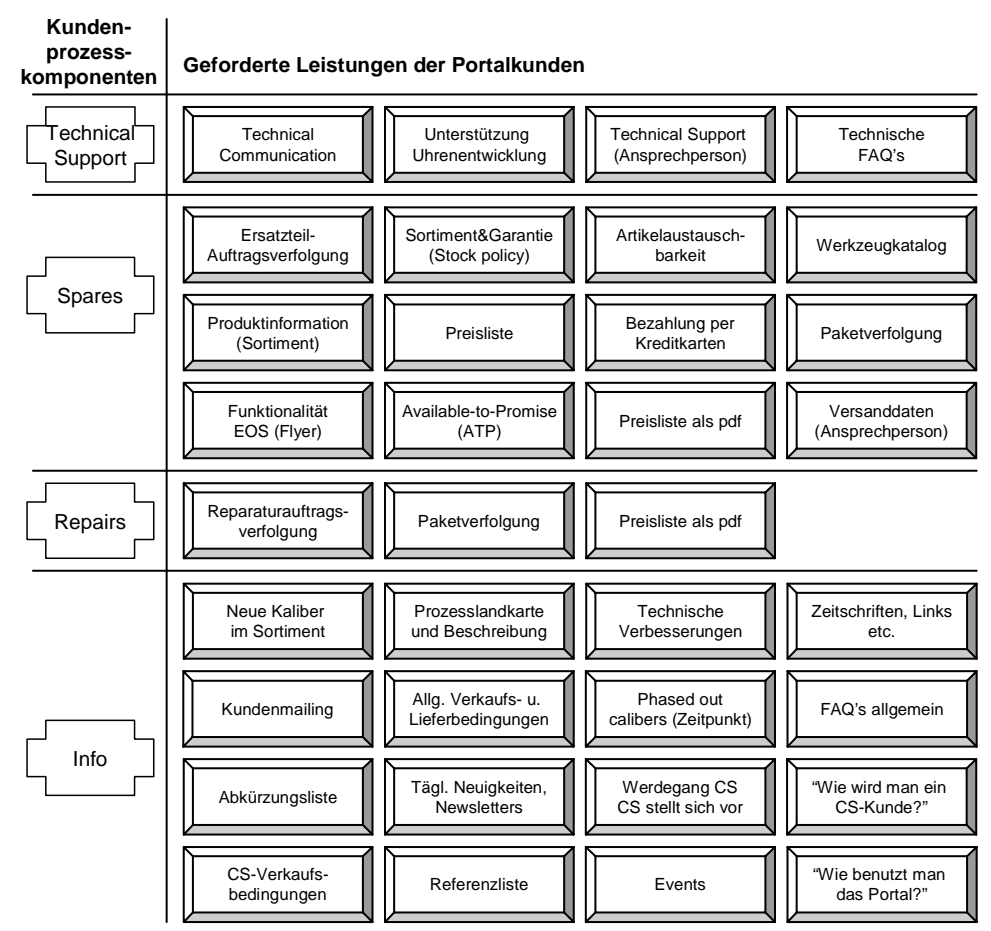

Bild 13-11: Kundenprozesskomponenten und geforderte Leistungen der Portalkunden

1. Untersuchungsbereich in 8 bis 10 Hauptprozesse zerlegen. Die Prozesse werden chronologisch gereiht. In den Workshops hat sich der ,typische Tagesablauf der Portalkunden' als Untersuchungsbereich bewährt. Die Prozesse werden anschliessend in einem Prozessverzeichnis beschrieben.

2. Hauptleistungen identifizieren und den Prozessen zuordnen. Leistungen, die von ,oben nach unten' fliessen werden links der Prozessreihe platziert. Rechts der Prozessreihe werden Leistungen platziert, die von , unten nach oben' fliessen. Jede Leistung erhält eine fortlaufende Nummer und wird in einem Leistungsverzeichnis beschrieben.

3. Zusätzliche Prozessbeteiligte oder Portalkunden als Leistungskonsumenten identifizieren. Weitere Portalkunden zu denen innerhalb der Prozesse Beziehungen existieren, werden in einer gesonderten Spalte im Leistungsverzeichnis erfasst und im Portalkundenverzeichnis beschrieben.

4. Bestehende Informationssysteme zur Unterstützung der Leistungserbringung identifizieren. Die IS werden in einer gesonderten Spalte im Leistungsverzeichnis erfasst und in einem Informationssystem-Verzeichnis beschrieben. 


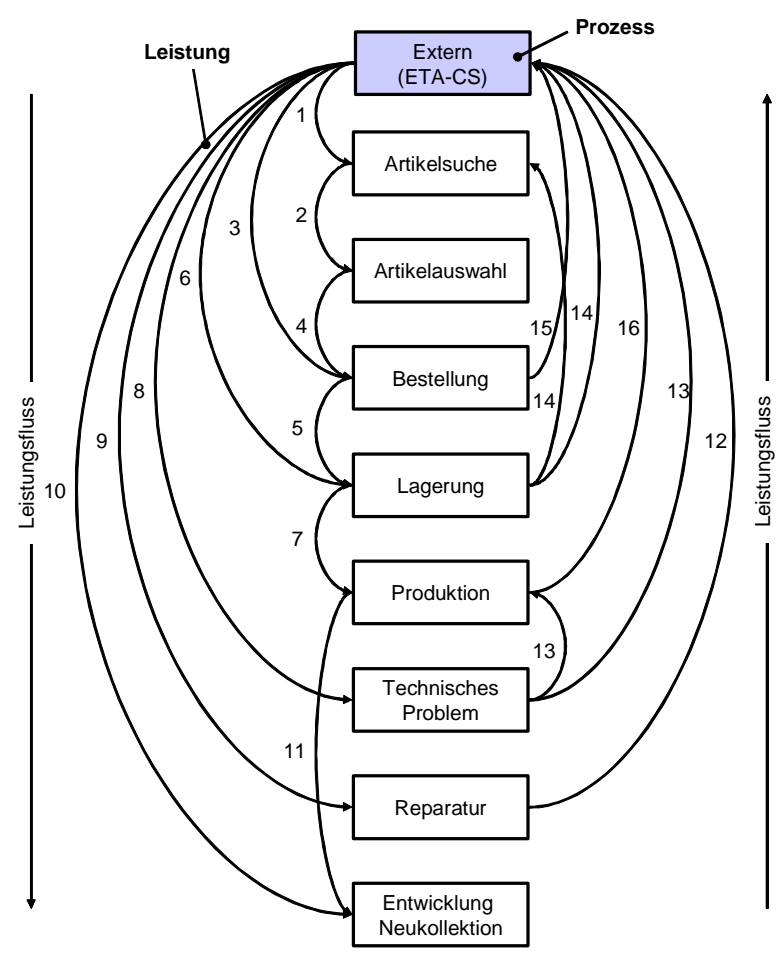

Bild 13-12: Kundenprozess-Matrix für ,Component Customer'

Endergebnis von Technik 2.1 sind die spezifizierten Kundenprozesse sowie die definierten Portalleistungen. Diese werden in einem ersten Schritt auf ihre Existenz überprüft. Kriterien dafür sind: ,elektronisch vorhanden', ,bisher nicht elektronisch vorhanden', ,bisher nicht vorhanden' sowie, nicht vorhanden erst für spätere Version(en) des Portals relevant'. Somit wird frühzeitig deutlich, welche Portalleistungen noch fehlen und ob und $\mathrm{zu}$ welchem Zeitpunkt sie im Projekt realisiert werden sollen.

Die Portalleistungen werden nach Abschnitten des Kundenprozesses (z.B. den Stufen des ,Customer Resource Life Cycle', s. Kap. 2.3.1) in Obergruppen eingeteilt. Bei der Bildung der Portalkategorien ist es entscheidend, eine übersichtliche, intuitive und vor allem schnelle Benutzerführung zu berücksichtigen. Für das CSPortal wurden vier Portalkategorien und spezielle Subkategorien definiert. Beispielsweise enthält ,Products \& Services' alle Leistungen rund um die Kernprodukte des ETA-CS. Dazu zählen die Unterkategorien ,Technical Services', ,Spares Tracking', ,Repair Tracking', , Products \& Ordering' und ,Repair'.

Nicht jeder Nutzer soll ungehindert auf alle Daten zugreifen oder alle Prozesse ausführen können. Die Authentifizierung identifiziert jeden Nutzer eindeutig, und 
die Autorisierung teilt ihm entsprechende Rechte und Pflichten zu. ${ }^{55}$ Damit nicht jeder Nutzer individuell im System geführt werden muss, werden sie Rollen oder Nutzergruppen zugeordnet und mit Rechten und Pflichten ausgestattet [Balzert 2000, 907]. Für die Zugriffskontrolle auf Portalleistungen wird eine eigene Berechtigungsliste (s. Bild 13-13), bestehend aus drei Nutzergruppen ,Besteller', ,Mitarbeiter' und ,Interessent' entworfen. Dabei kann die Nutzergruppe ,Interessent' nur öffentliche Kategorien wie ,About CS', oder ,Help \& Contact' einsehen. Die Gruppe ,Mitarbeiter', der in der Regel Ersatzteil- und/oder Reparaturkunden des CS angehören, kann zusätzlich die Tracking-Applikationen aufrufen, während die Nutzergruppe ,Besteller' Ersatzteile über den EOS bestellen kann.

\begin{tabular}{|c|c|c|c|c|c|c|}
\hline \multicolumn{2}{|c|}{ Prozess } & \multicolumn{5}{|c|}{ Kernaufgabe } \\
\hline \multicolumn{2}{|c|}{ Artikelsuche } & \multicolumn{5}{|c|}{$\begin{array}{l}\text { Identifikation zu bestellender Uhrwerkersatzteile und Bestimmung der Artikel im } \\
\text { Sortiment des ETA-CS }\end{array}$} \\
\hline \multicolumn{2}{|c|}{ Artikelauswahl } & \multicolumn{5}{|c|}{ Auswahl der zu kaufenden Artikel, Anzahl und Wahl der Verpackungsart } \\
\hline \multicolumn{2}{|c|}{ Bestellung } & \multicolumn{5}{|c|}{ Prüfung der Einkaufs- und Zahlungskonditionen und Abschicken der Bestellung } \\
\hline \multicolumn{2}{|l|}{$\ldots$} & \multicolumn{5}{|l|}{$\ldots$} \\
\hline Nr. & & \multicolumn{3}{|c|}{ Leistungen } & $\begin{array}{c}\text { Zusätzliche } \\
\text { Prozessbeteiligte }\end{array}$ & IS \\
\hline 1 & \multicolumn{4}{|c|}{$\begin{array}{l}\text { Technische Informationen, Info Habillage, Artikelaustauschbar- } \\
\text { keit, Stock Policy }\end{array}$} & ETA-CS & A \\
\hline 2 & \multicolumn{4}{|c|}{ Fehlende Artikel, Bestellmenge } & & B \\
\hline 3 & \multicolumn{4}{|c|}{ Liefer- und Zahlungskonditionen } & ETA-CS & A \\
\hline$\ldots$ & \multicolumn{4}{|c|}{$\ldots$} & $\ldots$ & $\ldots$ \\
\hline IS & \multicolumn{2}{|c|}{$\begin{array}{l}\text { Unterstützte } \\
\text { Leistung }\end{array}$} & Bezeichnung & \multicolumn{3}{|c|}{ Beschreibung } \\
\hline A & \multicolumn{2}{|c|}{$1,3,4,6,8$} & ETA Online Shop (EOS) & \multicolumn{3}{|c|}{ E-Shop-Lösung des ETA-CS } \\
\hline B & \multicolumn{2}{|l|}{$2,5,7,11$} & Ramco & \multicolumn{3}{|l|}{ ERP-System } \\
\hline$\ldots$ & \multicolumn{2}{|l|}{$\ldots$} & $\ldots$ & \multicolumn{3}{|l|}{$\ldots$} \\
\hline
\end{tabular}

Tabelle 13-5: Prozess-, Leistungs- und IS-Verzeichnis von ,Component Customer'

Für das CS-Portal gilt wie für den EOS, dass die Nutzerdaten im ERP-System gepflegt und anschliessend übertragen werden. Somit gibt es ein klar führendes System und Daten werden nicht redundant gepflegt. Auch die Zuordnung der Nutzer zu den Portal-Nutzergruppen ist im ERP-System hinterlegt.

${ }^{55} \mathrm{Zu}$ Authentisierung und Autorisierung vgl. [Ahuja 1996, 21ff], [Bernstein et al. 1996, 168ff], [Amor 1999, 396ff] und [Chesher/Kaura 1998, 183ff]. 


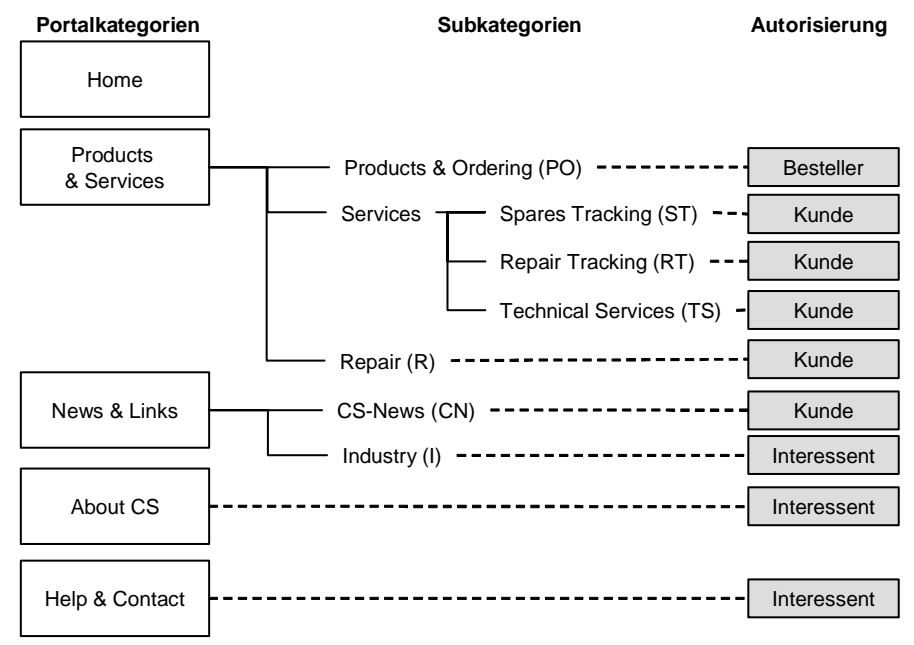

Bild 13-13: Portal-Berechtigungsliste

Die Repräsentation des Kundenprozesses entscheidet letztlich darüber, ob sich der Kunde im Portal zurechtfindet, es nutzt oder ob er sich mit seinen Problemen allein gelassen fühlt. Bei der Umsetzung ist zwischen zwei Extremen zu unterscheiden:

- Die strukturierte Linksammlung richtet die Kategorien am Kundenprozess aus und wendet sich an erfahrene Nutzer. Sie sind mit den Portalkategorien vertraut und gelangen so schnell zur gewünschten Leistung.

- Die vordefinierte Ablaufreihenfolge eignet sich für Einmal- oder Erstnutzer und gibt mit Fragen oder einem festen Workflow die zu durchlaufenden Aufgaben des Nutzers vor.

Linksammlungen wirken bei zunehmendem Leistungsangebot immer unübersichtlicher (beim ETA-CS wurden im ersten Workshop 59 Leistungen identifiziert). Hingegen ist die völligständig vordefinierte Ablaufreihenfolge bei der Nutzung von Einzelleistungen zeitintensiv und wenig benutzerfreundlich. Es empfiehlt sich daher, Ablaufreihenfolgen oder Workflows dort einzusetzen, wo sie zwingend erforderlich sind, etwa beim E-Shop und der Auftragsverfolgung. Linksammlungen sollten mit sprechenden Kategorien dort eingesetzt werden, wo das Ergebnis intuitiv erfassbar ist. Wie in vielen Portalen kommen beim ETA-CSPortal beide Formen zum Einsatz: Ablaufreihenfolgen in den Applikationen EOS, Spares, Parcel und Repair Tracking und Linksammlungen für die restlichen Leistungen. 


\subsubsection{Kooperationsprozessanalyse bei ETA SA}

Ausgehend vom Kundenprozess ermittelt Technik 2.2 die Potenziale eines Outtasking an Web Service-Anbieter. In einem ersten Schritt wird die Eignung der Aufgaben geprüft (z.B. vorhandene Kompetenz und Eignung einer Leistung für zeit- oder transaktionsbasierte Abrechnung). Im zweiten Schritt wird analysiert, wie spezifisch eine Leistung und wie hoch ihre strategische Bedeutung ist [Picot/Maier 1992]. Im dritten Schritt wird die zukünftige Strategie aufgrund der Ergebnisse aus Schritt 1 und 2 definiert. ETA-CS identifiziert fünf Leistungen als Outtasking-Kandidaten: Artikelverfügbarkeit, Kreditkartenzahlung, Paketverfolgung, branchenbezogene Informationen und Zeitschriften sowie Links. Der Untersuchungsbereich ,Intern/Kooperation' umfasst vier Leistungen: Technische Informationen, Lagervorschriften, Einbauanleitung und Entwicklungsunterstützung. Die anderen Bereiche wurden für eine spätere Untersuchung zurückgestellt.

\begin{tabular}{|c|c|c|c|c|c|c|c|}
\hline \multirow[b]{2}{*}{$\mathrm{Nr}$. } & & \multicolumn{3}{|c|}{ 1. Schritt } & \multicolumn{2}{|c|}{ 2. Schritt } & \multirow{2}{*}{$\begin{array}{l}\text { 3. Schritt } \\
\text { Künftige } \\
\text { Strategie }\end{array}$} \\
\hline & & 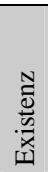 & 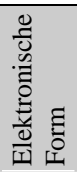 & 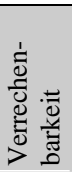 & 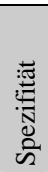 & 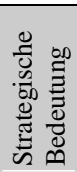 & \\
\hline 1 & $\begin{array}{l}\text { Technische Infos, Info Habillage, Lager- } \\
\text { verwaltungsstrategie, Artikelaustausch- } \\
\text { barkeit }\end{array}$ & & (1) & & & & Intern \\
\hline 2 & Liefer- und Zahlungskonditionen & C & O & $\bigcirc$ & 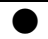 & (1) & Intern \\
\hline 3 & Individueller Warenkorb & $\bigcirc$ & $\bigcirc$ & O & (1) & D & Intern \\
\hline 4 & Auftragsverfolgung & $\bigcirc$ & $\bigcirc$ & & O & & $\begin{array}{l}\text { Intern, } \\
\text { überprüfen }\end{array}$ \\
\hline 5 & Lagervorschriften & 0 & $\bigcirc$ & $\bigcirc$ & & & Intern \\
\hline 6 & Artikelverfügbarkeit & $\bigcirc$ & $\bigcirc$ & & $\bigcirc$ & $\bigcirc$ & Outtasking \\
\hline 7 & Einbauanleitung, bekannte Probleme & & $\bigcirc$ & O & & & Kooperation \\
\hline 8 & Statusverfolgung für Reparaturen & $\bigcirc$ & $\bigcirc$ & & & & $\begin{array}{l}\text { Intern, } \\
\text { überprüfen }\end{array}$ \\
\hline 9 & Entwicklungsunterstützung für Uhren & & $\bigcirc$ & 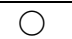 & & & Kooperation \\
\hline 10 & Produktionspläne und -kapazitäten & $\bigcirc$ & $\bigcirc$ & & & 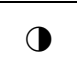 & $\begin{array}{l}\text { Intern, } \\
\text { überprüfen }\end{array}$ \\
\hline 11 & Defektes Werk, Beschwerdemanagement & $\bigcirc$ & $\bigcirc$ & $\bigcirc$ & & & Kooperation \\
\hline 12 & Bezahlung per Kreditkarten & $\bigcirc$ & $\bigcirc$ & & $\bigcirc$ & $\bigcirc$ & Outtasking \\
\hline 13 & Paketverfolgung & $\bigcirc$ & $\bigcirc$ & & $\bigcirc$ & $\bigcirc$ & Outtasking \\
\hline 14 & Branchenbezogene Informationen & $\bigcirc$ & $\bigcirc$ & & $\mathrm{O}$ & $\bigcirc$ & Outtasking \\
\hline 15 & Zeitschriften, Links etc. & $\bigcirc$ & $\bigcirc$ & & $\bigcirc$ & $\bigcirc$ & Outtasking \\
\hline
\end{tabular}

Legende: Gegeben Teilweise gegeben $\bigcirc$ Nicht gegeben

Tabelle 13-6: Beispiel einer Leistungsgapanalyse

Für die Leistungen, die sich für ein Outtasking eignen, wird eine Prozessarchitektur erstellt (s. Bild 13-14). Die Leistung ,Paketverfolgung' wird beispielsweise der Kundenprozesskomponente ,Track \& Trace', dem Hauptprozess ,Distribution' 
und dem Teilprozess ,Transport' zugeordnet. Distribution unterteilt sich in die Teilaufgaben Auftrags-, Zahlungs- sowie Transportabwicklung. Die Leistung ,Bezahlung per Kreditkarte' wird über die Kundenprozesskomponente ,Bezahlung' dem Teilprozess ,Zahlungsabwicklung' zugewiesen.

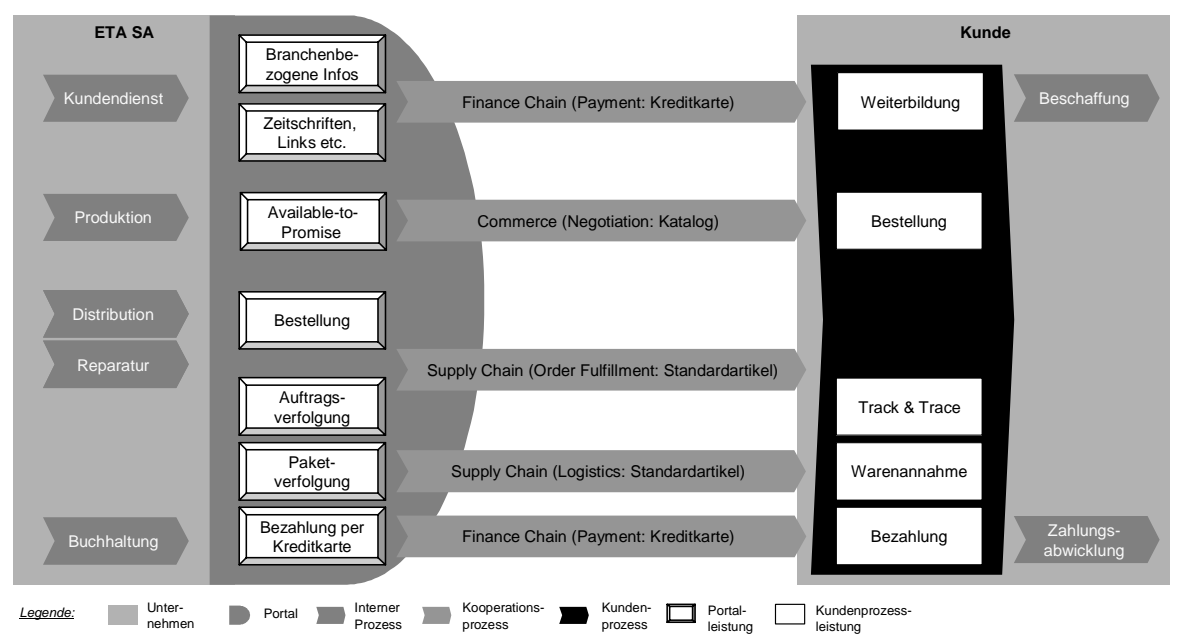

Bild 13-14: Prozessarchitektur-IST mit potenziellen Outtasking-Leistungen

Die Architektur enthält neben Kundenprozess und Portalleistungen auch die Kooperationsprozesse, welche die Leistungserbringung mit den beteiligten Unternehmen konkretisieren. Für das ETA-Portal steht der Auftragsabwicklungsprozess im Mittelpunkt. Die Aufgaben dieses Prozesses zeigt ein Aufgabenkettendiagramm (s. Bild 13-15). Anschliessend erfolgt eine Kernkompetenzanalyse, die sich an den drei Teilschritten der Leistungsgapanalyse orientiert (s. Tabelle 13-7).

Für den ETA-CS liessen sich drei Aufgaben für die Auslagerung an Web Service-Anbieter identifizieren: ,Plane und stelle Frachten zusammen und bestätige', ,Erstelle Versanddokumente' und ,Sende Auftragsstatus (Order tracking)'. Für diese Aufgaben werden die Ist-Kosten aus den Aufwendungen der Kostenstellen und aus den Bezugsgrössen der Leistungserbringung (Pakete, Aufträge und Anrufe) sowie die anfallenden Kosten je Bezugsgrösse aus dem Mitarbeitereinsatz ermittelt. Entfallen durch das Outtasking leistungsmengenneutrale Kosten [Wisskirchen/Mertens 1999, 294], sind diese miteinzubeziehen. Die Verrechnung der Kostenstellenkosten auf die Prozesskosten erfolgt der Einfachheit halber anteilig zu den ermittelten Mitarbeitertagen pro Jahr. Direkt einer Aufgabe zuordenbare Einzelaufwendungen, wie die Kosten einer Schachtel für die Aufgabe ,Stelle Paket zusammen', werden ebenfalls zu den aufgabenbezogenen Kosten addiert. Kostenstellenkosten von etwa CHF 1'000'000 werden auf die Bezugsgrössen umgelegt, so dass sich Kosten von CHF 21,60 je Paket, CHF 12,12 je Transport und CHF 10,23 je Anfrage ergeben. Ein Web Service, der Versanddokumente erstellt, sollte also nicht mehr als CHF 21,60 je Paket kosten. Neben den Kosten können in die Auswahl Marktinformationen und weitere Kriterien einfliessen. 


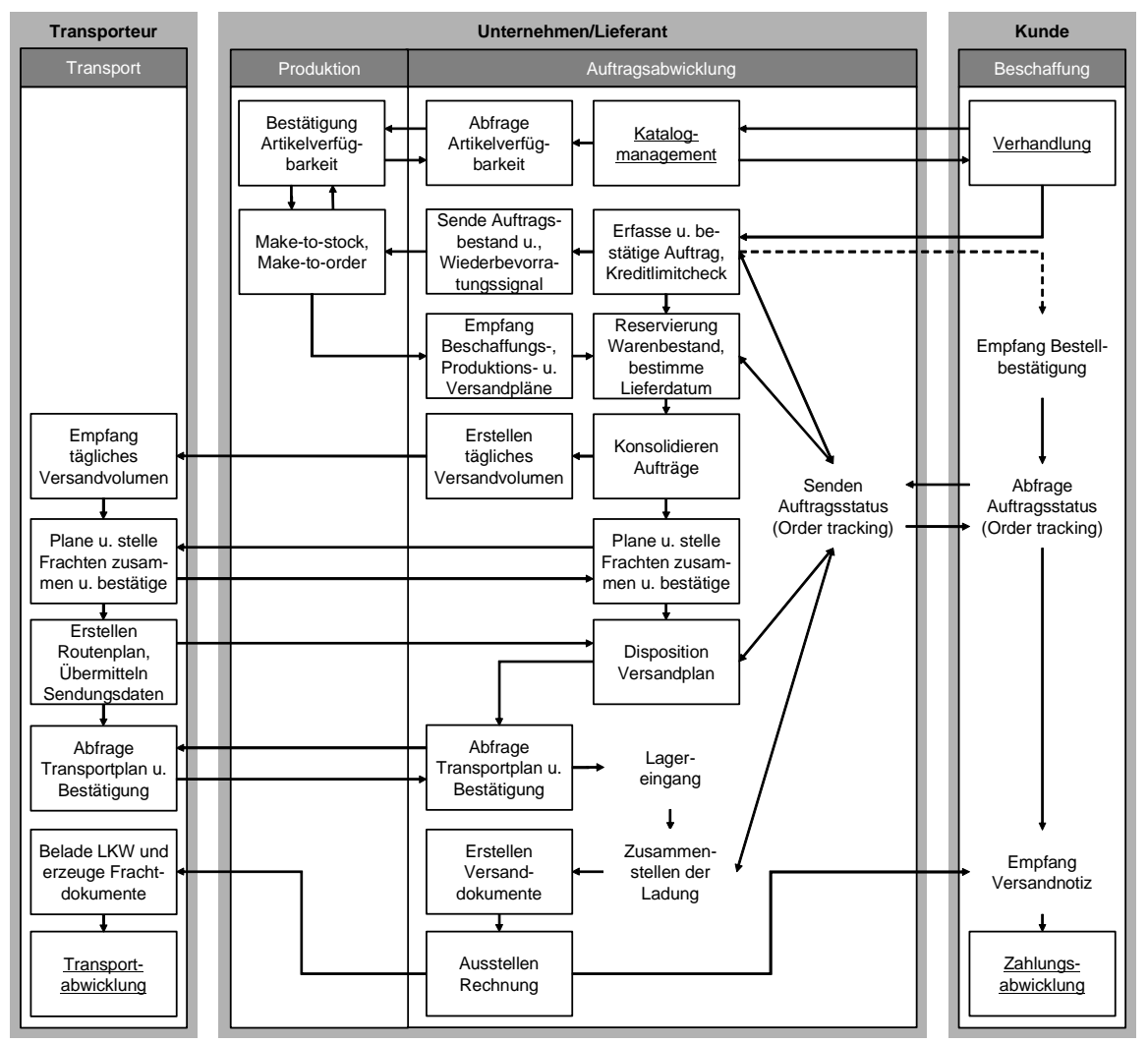

Bild 13-15: Aufgabenkettendiagramm der Ist-Auftragsabwicklung

Stehen die Web Service-Anbieter fest, lässt sich eine Soll-Prozessarchitektur entwerfen (s. Bild 13-16). Gemeinsam mit dem Web Service-Anbieter und Kunden werden die Soll-Aufgabenkettendiagramme entwickelt und die neuen bzw. optimierten Aufgaben und (modifizierten) Leistungen integriert. ETA-CS schliesslich hat die Aufgaben ,Erzeuge Versanddokumente' und ,Hole Angebote für tägliches Versandvolumen' an inet-Logistics ausgelagert. 


\begin{tabular}{|c|c|c|c|c|c|c|}
\hline \multirow[b]{3}{*}{ Nr. } & \multirow[b]{3}{*}{ Aufgaben } & \multirow[b]{3}{*}{ Ergebnis der Aufgaben } & \multicolumn{4}{|c|}{ Schritte } \\
\hline & & & 1 & & 2 & 3 \\
\hline & & & 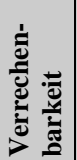 & 离 & 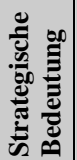 & $\begin{array}{l}\text { Zukünftige } \\
\text { Strategie }\end{array}$ \\
\hline 1 & $\begin{array}{l}\text { Frage Artikelverfüg- } \\
\text { barkeit ab }\end{array}$ & $\begin{array}{l}\text { Lagerbestand } \\
\text { Artikelverfügbarkeit }\end{array}$ & & $\bigcirc$ & & $\begin{array}{l}\text { Überprüfen, } \\
\text { intern }\end{array}$ \\
\hline 2 & $\begin{array}{l}\text { Bestätige Artikelver- } \\
\text { fügbarkeit }\end{array}$ & $\begin{array}{l}\text { Lagerbestand } \\
\text { Artikelverfügbarkeit }\end{array}$ & & 0 & & $\begin{array}{l}\text { Überprüfen, } \\
\text { intern }\end{array}$ \\
\hline 3 & $\begin{array}{l}\text { Erfasse, bestätige } \\
\text { Auftrag, überprüfe } \\
\text { Kreditlimit }\end{array}$ & $\begin{array}{l}\text { Auftrag } \\
\text { Auftragsbestätigung } \\
\text { Kreditlimit }\end{array}$ & & $\bigcirc$ & & $\begin{array}{l}\text { Überprüfen, } \\
\text { intern }\end{array}$ \\
\hline 4 & $\begin{array}{l}\text { Sende Auftragsbestand } \\
\text { und Wiederbevorra- } \\
\text { tungssignal }\end{array}$ & $\begin{array}{l}\text { Auftragsbestand } \\
\text { Lagerbestand } \\
\text { Wiederbevorratungssignal }\end{array}$ & ) & $\bigcirc$ & & $\begin{array}{l}\text { Intern/ } \\
\text { Kooperation }\end{array}$ \\
\hline 5 & $\begin{array}{l}\text { Make-to-stock, } \\
\text { Make-to-order }\end{array}$ & $\begin{array}{l}\text { Produktionsauslastung } \\
\text { Produktionsmenge }\end{array}$ & & & & $\begin{array}{l}\text { Intern/ } \\
\text { Kooperation }\end{array}$ \\
\hline 6 & $\begin{array}{l}\text { Reserviere Warenbe- } \\
\text { stand, bestimme Lie- } \\
\text { ferdatum }\end{array}$ & $\begin{array}{l}\text { Bestandsreservierung } \\
\text { Lieferdatum }\end{array}$ & a & () & (1) & Überprüfen \\
\hline 7 & $\begin{array}{l}\text { Erhalte Beschaffungs-, } \\
\text { Produktions- und } \\
\text { Versandpläne }\end{array}$ & $\begin{array}{l}\text { Beschaffungsplan } \\
\text { Produktionsplan } \\
\text { Versandplan }\end{array}$ & C & & & $\begin{array}{l}\text { Intern/ } \\
\text { Kooperation }\end{array}$ \\
\hline 8 & Konsolidiere Aufträge & Konsolidierter Auftragsbestand & $\bigcirc$ & & & $\begin{array}{l}\text { Intern/ } \\
\text { Kooperation }\end{array}$ \\
\hline 9 & $\begin{array}{l}\text { Plane und stelle Frach- } \\
\text { ten zusammen und } \\
\text { bestätige }\end{array}$ & Frachtplan & & 0 & $\bigcirc$ & Outtasking \\
\hline 10 & $\begin{array}{l}\text { Erzeuge Versanddoku- } \\
\text { mente }\end{array}$ & Versanddokumente & & $\Omega$ & 0 & Outtasking \\
\hline 11 & $\begin{array}{l}\text { Sende Auftragsstatus } \\
\text { (Order tracking) }\end{array}$ & Auftragsstatus & & $\frown$ & 0 & Outtasking \\
\hline
\end{tabular}

Legende: $\bigcirc$ Hoch Mittel/eher nicht $\bigcirc$ Gering/nicht möglich

Tabelle 13-7: Aufgaben-/Leistungsverzeichnis 


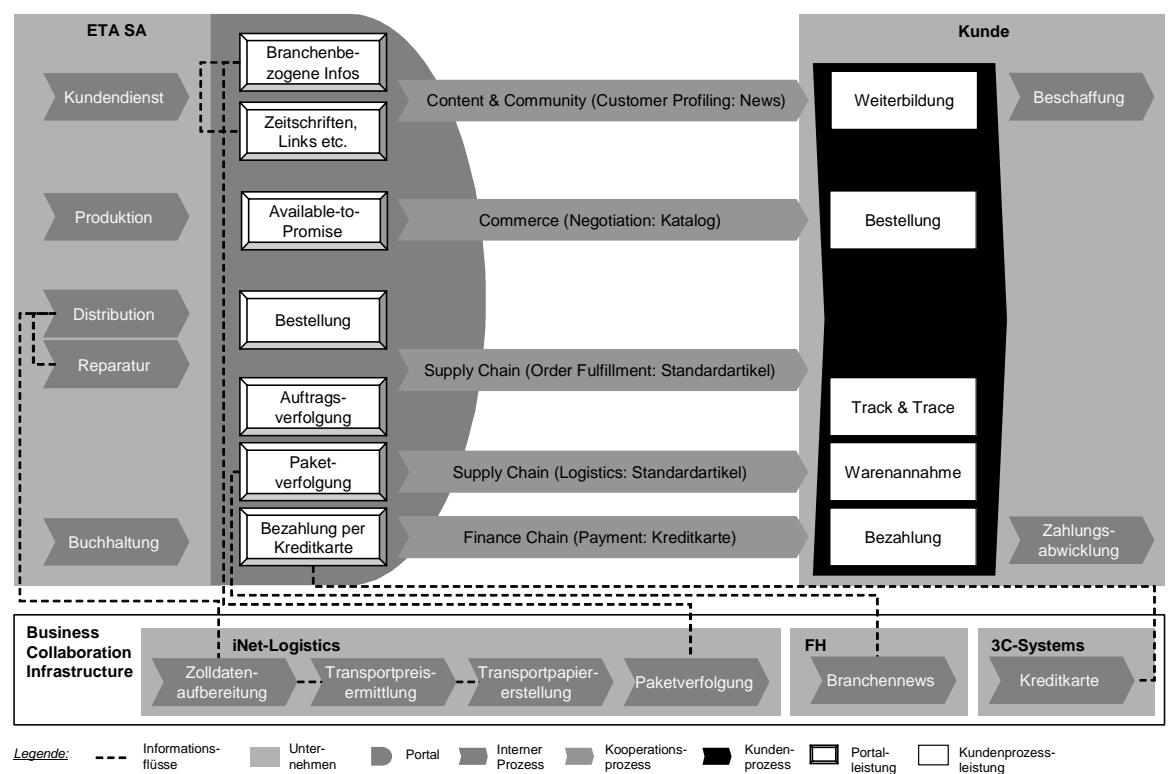

Bild 13-16: Soll-Prozessarchitektur mit Outtasking-Leistungen

\subsection{Zusammenfassung und Ausblick}

Die beschriebene Methode fasst die ersten Erfahrungen bei der Umsetzung von Prozessportalen zusammen. Diese sind das ,Front-end' des Echtzeitunternehmens und stellen in hoch individualisierter Form die vom Kunden benötigten Dienstleistungen zusammen. Die Umsetzung dieser einfach darstellbaren Vision ist keinesfalls trivial, sind doch umfassende Integrations- und AutomatisierungsAnstrengungen ,im Hintergrund' notwendig. Besteht diese integrierte Basis nicht, mag zwar eine gut gestaltete Benutzerschnittstelle vorliegen. Durch die geringe Aktualität der enthaltenen Informationen kann jedoch nicht von ,Real-time Business' gesprochen werden.

Zur Gestaltung von Echtzeit-fähigen Prozessportalen ist daher eine systematische Methodik notwendig, die zunächst anhand des Geschäftsnetzwerks und der Kundensegmente in der Portalstrategie die grundsätzliche Lösung ableitet und grob bewertet. Das Portaldesign leitet detailliert daraus den Kundenprozess sowie die Portalleistungen und spezifiziert die Kooperationsprozesse mit den beteiligten Web Services. Während das vorliegende Kapitel diese Schritte mit beispielhaften Ergebnisdokumenten illustriert hat, sei für die Ebene ,Portalsystem' auf die Kapitel 2 und 10 verwiesen. Erst über eine Integrationsarchitektur verbundene Applikationen stellen die Echtzeitanbindung der internen und externen Leistungen her. ,Real-time Business' bedeutet daher nicht zu unterschätzende Integrationsaufwendungen, denn von einem , plug\&play' der Prozesse und Systeme kann heute trotz 
der Marketingaussagen vieler Softwareanbieter nicht gesprochen werden (s. Kap. 7).

Ebenso wie die Prozess- und Datenstandards befinden sich noch viele Elemente des ,Real-time Business' im Entstehen. Beispiele sind die übergreifenden Lösungen von Softwareanbietern (s. Kap. 4), die standardisierten Web Services im Zahlungs- und Logistikbereich (s. Kap. 3, 4) sowie die sich zu Collaboration- bzw. Integrationsplattformen weiterentwickelnden elektronischen Marktplätze (s. Kap. 5). Wie auch Kapitel 6 gezeigt hat, müssen Unternehmen gegenwärtig noch viel Eigenarbeit in die Definition ihrer Echtzeit-Architekturen investieren. Allerdings sind diese ersten Schritte zum Aufbau von Erfahrungen mit den neuen Technologien notwendig. Dies gilt natürlich auch für den Einsatz intelligenter Geräte und Güter, die eine weitgehende Automatisierung am ,Point of Creation' (POC) und ,Point of Action' (POA) bewirken. Hier stehen die Lösungen in der Praxis noch am Anfang und werden verstärkt als Forschungsgegenstand erkannt und bearbeitet. Obgleich sich die Auswirkungen auf die Geschäftsprozesse heute noch nicht umfänglich abschätzen lassen, werden diese Technologien mit den in diesem Buch skizzierten Elementen des Echtzeitunternehmens (Kooperationsprozesse, Prozessportal etc.) eng integriert sein. Der Stellenwert von Architekturen und systematischer Methoden dürfte durch diese Entwicklungen aus heutiger Sicht daher deutlich zunehmen.

Zusammenfassend heisst Real-time Business für Unternehmen daher:

- Ihr Geschäftsnetzwerk in enger Abstimmung mit der Unternehmensstrategie an unterschiedlichen Kundensegmenten auszurichten. Daraus leiten sich die Leistungen ab, die das Unternehmen gemeinsam mit seinen Partnern anbieten kann. $\mathrm{Zu}$ den Partnern zählen neben den Lieferanten eine Vielzahl elektronischer Dienstleister.

- Den Kundenprozess für jedes Kundensegment detailliert zu analysieren und daraus die Leistungen zu erkennen, die in Echtzeit anzubieten sind. Für jede dieser Leistungen ist der Prozess vom Ort der Verwendung (POA) rückwärts zum Ort der Entstehung (POC) auf Medienbrüche und Informationspuffer zu untersuchen.

- Die einzelnen Aufgaben in den Kooperationsprozessen auf ihr ,Out-taskingPotenzial' zu überprüfen. Die daraus entstehende, Collaboration Infrastructure' kann entweder selbst zusammengestellt werden oder ein externer Anbieter übernimmt diese Aufgabe.

- Die Prozess- und Systemintegration als Schlüsselaufgabe zu erkennen. Übergreifende Standards und Architekturen reduzieren dabei die Spezifität der Echtzeit-Integration und entstehen in zunehmendem Masse. Hier gilt es die Entwicklungen zu verfolgen und bereits heute erste Erfahrungen zu sammeln.

- Eine Vision in kleinen, wohlüberlegten Schitten umzusetzen. Mit den in diesem Buch vorgestellten Modellen lassen sich verschiedene Zielzustände abbilden, die bei der sukzessiven Entwicklung einer längerfristigen kundenorientierten Strategie helfen sollen. Alleine die hohe Integrationskomplexität zwischen mehreren Partnern verbietet aber die Realisierung mit einem ,grossen Wurf'. 